\title{
Plateforme de prototypage rapide d'objets connectés avec la famille WiNo*
}

\section{Enabling Fast-prototyping of Connected Things using the WiNo* family}

\author{
Adrien van den Bossche ${ }^{1}$, Réjane Dalcé ${ }^{2}$, Thierry Val ${ }^{1}$ \\ ${ }^{1}$ IRIT, Université de Toulouse, \{vandenbo,val\}@irit.fr \\ ${ }^{2}$ IRIT, INU Champollion, dalce@irit.fr
}

\begin{abstract}
RÉSUMÉ. Avant d'être déployés sur le terrain, les différentes solutions constituant le réseau de collecte de l'Internet des Objets (Device-Layer - Internet of Things) doivent être évaluées en termes de performances, de passage à l'échelle et de reproductibilité. Dans cet article, nous présentons notre plateforme de test, conçue pour permettre d'atteindre ces différents objectifs. Cette plateforme, basée sur toute une famille de nœuds, est présentée ainsi que des exemples d'exploitation de ses capacités.

ABSTRACT. In order to be able to deploy the various solutions for the Device Layer of the Internet of Things, these proposals must be evaluated in terms of performance, scalability and repeatability. This paper introduces our test platform which was designed to be able to support these types of tests. We describe the wireless nodes which make up the test platform and present a selection of use cases.

MOTS-CLÉS. IdO, prototypage rapide, plateforme, Arduino, WiNo, Teensy, DL-loT, nœud.

KEYWORDS. IOT, fast prototyping, platform, Arduino, WiNo, Teensy, DL-loT, node.
\end{abstract}

\section{Introduction}

L'Internet des objets (Internet of Things, IoT) est en train de révolutionner le domaine des réseaux et des télécommunications. De nombreux spécialistes prévoient, pour les années à venir, une explosion du nombre des objets connectés [SHA 17] dans les pays industrialisés, en particulier dans le contexte d'applications liées au confort, aux loisirs, à la qualité de vie et à la santé. Les pays émergents sont également susceptibles de profiter de ce bouleversement en raison de l'existence de nombreuses applications générant des besoins forts (agriculture raisonnée, énergies renouvelables...). Cette possibilité est renforcée par la simplicité de déploiement des réseaux sans fil en zones désertiques ou hostiles. Parmi ces objets connectés se trouvent des éléments fixes, monitorant l'environnement, et des éléments mobiles, portés par un humain, attachés à du bétail ou encore à des véhicules intelligents. Aujourd'hui, ces différentes classes forment des réseaux séparés car utilisant des protocoles de communication et des technologies souvent non interopérables. L'objectif de l'IoT est de les faire collaborer en les dotant tous de la capacité de communiquer via Internet. Cette révolution se situe à plusieurs niveaux de compétences et ouvre de grandes opportunités de contributions. Elle est associée au concept de communications de Machine à Machine, M2M, où de nombreux travaux sont en cours [JAE 14]. Les aspects plus matériels et les couches basses, qui constituent le réseau de collecte (DL-IoT - Device Layer of the IoT) -premier ou dernier maillon dans la structure globale de 1'IoT-, trouvent aussi dans ce «Cloud des objets» une seconde jeunesse et des axes de recherche pour les spécialités des réseaux de capteurs sans fil (Wireless Sensor Networks, WSN). Les outils et méthodes de développement des réseaux et des protocoles doivent eux aussi être adaptés à l'IoT. Nous constatons, à l'heure actuelle, l'émergence de nombreux travaux [LEE 16] se basant sur des outils de simulation comme par exemple NS3 [NS3 17] ou Cooja pour Contiki, pour tester et analyser les nouvelles couches basses du réseau de collecte. Néanmoins, la grande évolution de ces dernières années reste, en parallèle et en complément à ces simulateurs, l'orientation marquée de la communauté scientifique vers le prototypage et l'analyse de performances réelles permise par des plateformes de test matérielles, que l'on désigne par testbed. Le faible coût économique et la grande disponibilité de composants efficaces 
et totalement reprogrammables favorisent le développement de ces techniques. Plusieurs nouvelles plateformes voient le jour et sont souvent issues d'environnements mis en place il y a quelques années pour les WSN.

Nos travaux se situent dans ce contexte et contribuent à la proposition et à la mise à disposition d'une plateforme ouverte pour le prototypage rapide de nouds pouvant être utilisés pour des objets connectés. L'un des choix stratégiques a été d'inscrire notre plateforme dans le paysage du libre, tant du point de vue du matériel que du logiciel. Nos contributions se distinguent de la concurrence par l'utilisation de composants du monde Arduino, la capacité à intégrer un grand nombre de transceivers et donc, une très grande diversité de couches physiques existantes et à venir, et une ergonomie facilitant le développement de protocoles et d'applications innovants.

Après cette introduction, nous débuterons cet article par une présentation du contexte normatif « Réseaux et Protocoles » de l'IoT. Nous présenterons ensuite dans le chapitre 3 un état de l'art les principales plateformes existantes en vue de faire aussi bien du prototypage et de l'évaluation des performances des protocoles, que de l'évaluation par les usages. Nous détaillerons ensuite dans le chapitre 4 notre modèle d'architecture, désigné par «WiNo », ainsi que les différentes déclinaisons de ce modèle en plateforme d'évaluation, offrant notamment une grande diversité de couches physiques. Dans le chapitre 5, nous illustrerons leurs utilisations sur quelques exemples typiques, avant de conclure et présenter les perspectives de nos travaux.

\section{Contexte}

Depuis le début des années 2010, le domaine des réseaux personnels et locaux sans fil (Wireless Personnal Area Network, WPAN et Wireless Local Area Network, WLAN) voit l'émergence de nombreux modes de transmission en rupture avec l'existant. De nombreuses technologies de couche physique (PHY) et les couches d'accès au médium (MAC) associées, propriétaires ou issues de standards ouverts, sont désormais communément rencontrées et implémentées dans les appareils. Cette situation rappelle la bataille des standards menée dans les années 1990, qui allait déboucher sur un standard unique, permettant d'unifier toutes les communications sans fil de courte distance, aussi bien WLAN que WPAN. La meilleure illustration de ce concept de protocole unifié est Bluetooth, qui, à l'aube du 21e siècle, avait gagné la bataille des standards des WPAN, avec Wifi et Zigbee - tous trois issus de la sphère IEEE. Dix ans plus tard, les conditions technologiques et économiques ont permis à nombre de protocoles de transmission innovants de voir le jour, ceci aussi bien dans les modes de transmission en rupture (par la lumière : LiFi [lifi] ou par l'Ultra Wide Band, UWB) que les protocoles propriétaires souhaitant s'établir comme des standards (Ant+, LoRaWAN). Les protocoles issus de la sphère IEEE s'inscrivent eux aussi dans cette évolution : de nombreuses extensions des protocoles existants sont proposées (802.11ac, 802.15.1 BLE, 802.15.4-2011 en CSS et UWB, 802.15.4-2012 pour l'industrie) et de nouveaux standards proposés (802.15.6). Enfin, les champs très ouverts de la radio cognitive (cognitive radio) ou de la radio logicielle (Software Defined Radio, SDR, Software Defined Network, SDN) parachèvent le coup de pied donné dans la fourmilière et rebattent encore un peu plus les cartes. Nous assistons bel et bien à une explosion des diversités où le standard unique semble encore une fois un concept utopique et bien éloigné de la réalité du terrain.

Mais ce qui s'annonçait comme une nouvelle bataille ne l'est peut-être pas, en réalité : d'une part, la diversité des modes de transmission semble assumée tant par les concepteurs que par et les utilisateurs ; d'autre part, la disponibilité d'IPv4 et IPv6, y compris sur des cibles matérielles très limitées en ressources, garantit la convergence au-dessus de couches physiques hétérogènes. Les avancées technologiques et économiques en termes d'intégration électronique sont telles que les objets communicants intègrent aujourd'hui plusieurs technologies de couches basses sans difficulté majeure, ces dernières finissant par converger grâce aux couches hautes. Le meilleur exemple en est la tablette ou le smartphone, qui intègre depuis des années un nombre important de technologies de communication différentes : $2 \mathrm{G} / 3 \mathrm{G} / 4 \mathrm{G}$, WiFi, Bluetooth, NFC, et bientôt LTE/5G et NB-IoT 
(NarrowBand-IoT). Enfin, le software empiétant sur le hardware, il suffit parfois d'installer une application pour rendre compatible un appareil avec un nouveau mode de transmission; ce dernier point pourrait bien se généraliser dans le futur avec la démocratisation de la SDR. Les années à venir diront si le marché, dans sa globalité, accepte définitivement cette diversité.

Nous avons décidé de parier sur la coexistence de différents modes de transmission plutôt que sur une technologie radio unique. Par conséquent, l'objectif principal de nos travaux est de fournir une architecture matérielle et logicielle permettant l'exploitation de ces différents modes de transmission, via ces différentes couches physiques, en conservant un couplage entre les protocoles étudiés et les technologies de communication utilisées, dans une approche de prototypage rapide, en vue d'aller rapidement vers des jeux de résultats pertinents en situation d'usage. Cette approche permet de faciliter l'intégration de nouvelles solutions mais aussi de choisir la meilleure couche physique pour la pile protocolaire sélectionnée et les applicatifs variés. Dans la section 3, nous examinerons les plateformes existantes de prototypage rapide pour l'IoT et analyserons la manière dont elles adressent ce problème.

\section{Etat de l'art}

La problématique de l'IoT peut être abordée sous un angle de données comme sous un angle infrastructure. Dans le premier cas, la problématique concerne le traitement et la transformation d'une masse imposante de données en informations pertinentes (notion de BigData). En effet, les nœuds de ce réseau capturent des informations de l'environnement qui peuvent aller des caractéristiques classiques comme la température et la luminosité à la fréquentation d'un site ou l'intensité du champ magnétique. Regrouper les sources pour corréler les informations peut donner le jour à des applications novatrices. De même, dans ce contexte de crowdsourcing, s'assurer que les données observées sont les plus appropriées est un autre défi. Le développement d'un middleware devient l'enjeu principal puisqu'il rend transparentes les différences matérielles dans la mise en relation avec l'environnement. La plateforme Vital IoT [VIT 17] en est un exemple.

Approcher l'IoT avec une vision axée sur l'infrastructure emmène autant à développer des protocoles permettant l'interaction sécurisée et à distance avec des équipements, que la mise en place de structures permettant l'étude et le test de ces solutions. De par notre spécialisation en réseaux sans fil, nous nous intéresserons plutôt aux aspects communication de la problématique de l'Internet des Objets. La suite de cette section présentera donc un panorama des plateformes actuellement disponibles, ce qui nous permettra de positionner clairement notre contribution. Nous présenterons d'un côté quelques testbeds représentatifs en les examinant du point de vue de la diversité au niveau des couches basses, de la taille du réseau, du support de la mobilité et des outils de développement proposés. Nous proposons également un tour d'horizon de plateformes utilisables pour la mise en place d'un testbed local.

Nous assistons depuis quelques années à l'éclosion de plusieurs plateformes de développement, de tests réels et d'analyse de performances dédiées historiquement aux réseaux de capteurs sans fil et, actuellement de façon plus générale, à l'Internet des Objets. Les nœuds composant ces plateformes sont souvent équipés de systèmes d'exploitation légers et adaptés. TinyOS a été l'un des premiers systèmes d'exploitation open source conçus spécifiquement pour les systèmes embarqués communicants légers, et associés à des outils de développement et de simulation. D'autres plateformes logicielles et matérielles existent comme RIOT [RIO 17], LiteOS [CAO 08], Contiki [DUN 04], développé en particulier pour des microcontrôleurs TI MSP430 et Atmel AVR. Les Motes TelosB, Tmote ou MicaZ sont basées sur cet environnement logiciel. Les testbeds que nous décrirons par la suite sont construits principalement au-dessus de ces différents nœuds.

Plus proches de nous, des projets de recherche ont permis de concevoir des environnements complets, accessibles aux utilisateurs distants. On parle communément de bancs de test pour désigner ces plateformes comportant un très grand nombre de nœuds, ouvertes et accessibles à la demande. Ces 
plateformes permettent à des utilisateurs de tester leurs protocoles, de la validation fonctionnelle à l'analyse des performances, sur un nombre restreint de nœuds comme en situation de passage à l'échelle. L'un des premiers testbeds publics en France a été SensLab [sens], de l'INRIA, soutenu initialement par l'agence française pour la recherche (ANR). Marqueur de l'évolution généralisée des réseaux de capteurs sans fil vers l'Internet des Objets, Senslab a évolué en 2012, en donnant naissance à la plateforme FIT/IoTlab [FLE 15]. Il faut également remarquer l'existence d'équipes de recherche entièrement axées vers la proposition de plateformes dédiées au testbed de réseaux de capteurs sans fil et d'objets communicants, comme par exemple Planet [KET 14], NITOS et Orbit [ORB 17].

La plateforme SmartSantander pour les villes intelligentes rivalise avec FIT/IoT-LAB en ce qui concerne l'ampleur du déploiement : chacun de ces testbeds met à disposition plusieurs milliers de nœuds. INDRIYA et TWIST donnent chacune accès à près de 200 nœuds, déployés sur plusieurs étages. Les nœuds de FIT/IoT-LAB communiquent via le standard IEEE 802.15.4-2006, à 860MHz et 2.4GHz. Dans le cas de SmartSantander, l'hétérogénéité des plateformes matérielles et des mécanismes de communication est forte car la population du réseau comprend des tags NFC, des smartphones, de nœuds embarqués sur les bus de transport public, etc. Cette diversité est un facteur permettant à la plateforme de se rapprocher de la situation réelle d'interaction de nœuds dotés de technologies hétérogènes. Bien que nous nous concentrions sur une diversité au niveau communication, il ne faut pas négliger la variété au niveau des capteurs intégrés aux nœuds. Ainsi, les nœuds de FIT/IoT-LAB comportent des accéléromètres, des magnétomètres et des gyromètres. La plateforme INDRIYA, quant à elle, n'utilise qu'une seule technologie radio mais propose des capteurs de luminosité, de rayonnement infrarouge, des magnétomètres, des accéléromètres et des capteurs acoustiques. Elle a aussi la particularité de disposer d'un cluster de nœuds Arduino. SmartSantander et FIT/IoT-LAB intègrent également la composante mobilité. En effet, l'IoT comporte autant des nœuds fixes que des nœuds en déplacement, tout simplement car attachés à un humain (son Smartphone par exemple). Smartsantander le met en œuvre en incorporant des smartphones à son réseau ainsi que des bus, alors qu'en indoor, la plateforme FIT/IoT-LAB se base sur des robots.

Concernant les aspects logiciels, il ressort de nos lectures que certains dispositifs sont devenus pratiquement des standards : par exemple, disposer d'une interface web pour la visualisation des résultats avec des granularités variables, pouvoir reprogrammer les nœuds à distance, générer des traces d'exécution mais également collecter des données de contexte comme des courbes de consommation énergétique durant l'expérience. Ces informations sont généralement produites par un réseau parallèle : il permet une communication hors-bande afin de ne pas affecter les résultats de l'expérience en cours. Dans le cas de FIT/IoT-LAB, les données sont stockées dans des fichiers CSV alors que SmartSantander et INDRIYA les stockent dans une base de données. Certains testbeds offrent des fonctionnalités additionnelles : par exemple, TWIST permet de tester sans la coopération du nœud la mort d'un élément de réseau en pilotant l'activation de l'interface USB utilisée pour l'alimentation.

Les outils recommandés pour une pré-évaluation du code à soumettre et les systèmes d'exploitation embarqués diffèrent d'une plateforme à l'autre. INDRIYA et TWIST utilisent TinyOS mais le premier propose TOSSIM alors que le second recommande d'utiliser Cooja, un simulateur disposant d'un plugin permettant de passer facilement de la simulation au testbed. Dans le cas de FIT/IoT-LAB, différents OS sont supportés (FreeRTOS, Contiki, RIOT, TinyOS, OpenWSN) et le choix du simulateur est laissé à la discrétion de l'utilisateur.

Après cet aperçu de testbeds existants pour l'IoT ainsi que de certains critères de comparaison, nous allons plonger dans le détail de ces plateformes matérielles adaptées à la mise en œuvre d'un testbed personnel. Nous les examinerons du point de vue de la facilité de réplication mais aussi du support explicite de couches physiques multiples. En effet, le fait de mener sa recherche en utilisant une plateforme de test pouvant être mise en place par une autre équipe facilite les contrôles de reproductibilité. 
En considérant la reproductibilité, l'intérêt que représente l'utilisation des composants de type Arduino comme élément matériel de base pour ces testbeds devient évident. D'une part, la plateforme tend alors à intégrer le caractère ouvert de l'écosystème Arduino, tant sur le plan matériel que logiciel. En effet, l'Open Source ayant montré son efficacité dans le domaine du software, le hardware en adopte aujourd'hui les codes (le design des cartes sous licence BSD, Creative Commons voire même GPL, etc.). La communauté peut alors s'emparer de ces systèmes et faire progresser de manière efficiente l'innovation dans le domaine de l'Internet des Objets. D'autre part, au-delà des protocoles et des aspects « réseau » à proprement parler, la grande accessibilité de ces plateformes, tant sur le plan technique qu'économique, fait que leur usage se répand en dehors du cadre de la communauté Télécoms et Réseaux : dans la communauté en Interaction Homme-Machine (IHM), des expérimentations sont menées sur les usages [DUC 15], [VEL 13] ; dans la communauté eHealth, des prototypes [VIG 15], [WU 08], [SUR 99], [ABD 17] sont développés avec des capteurs réels, etc. Cette pluridisciplinarité permet de faire émerger des produits et services innovants, qui vont bien au-delà de la discipline des Télécoms et Réseaux. Ces dispositifs, orientés prototypage rapide, permettent d'élaborer rapidement des systèmes présentant un Technology Readiness Level, TRL suffisamment élevé (6-7) pour convaincre de potentiels investisseurs, par exemple dans une approche de financement participatif.

Comme on peut le constater à la lecture de ce tour d'horizon rapide, de nouvelles plateformes émergent de manière très variée. Les solutions d'évaluation des performances sont très diverses et tendent vers le pragmatisme, en privilégiant l'analyse des performances réelles plutôt que celles uniquement simulées, et les performances dans leur globalité, c'est-à-dire en incluant le système, les capteurs et même l'usage, plutôt que de se cantonner aux critères de performance habituels (débit, latence, perte de messages, etc.).

\begin{tabular}{|c|c|c|}
\hline & $\begin{array}{c}\text { FIT/IoT-LAB } \\
2782 \text { nouds sur } 8 \text { sites }\end{array}$ & $\begin{array}{c}\text { SmartSantander } \\
20000 \text { nœuds prévus sur } 4 \text { sites }\end{array}$ \\
\hline Outils de gestion et interaction & $\begin{array}{l}\text { Client web et outils python en ligne de } \\
\text { commande: contrôle de l'état et de la } \\
\text { configuration des nœuds, réservation de } \\
\text { ressources et contrôle de l'exécution du } \\
\text { scénario }\end{array}$ & $\begin{array}{l}\text { Client web et scripts shell : réservation } \\
\text { de ressources et contrôle d'expérience }\end{array}$ \\
\hline Prise en main & $\begin{array}{l}\text { Multiples tutoriels disponibles Accès } \\
\text { possible à la plateforme }\end{array}$ & $\begin{array}{l}\text { Tutoriel disponible Enregistrement en } \\
\text { ligne à la plateforme indisponible }\end{array}$ \\
\hline Diversité des technologies radio & $\begin{array}{c}\text { IEEE } 802.15 .4(2.4 \mathrm{GHz}) \text { IEEE } 802.11 \\
\mathrm{a} / \mathrm{b} / \mathrm{g}\end{array}$ & GPRS IEEE 802.15.4 (2.4GHz) \\
\hline $\begin{array}{c}\text { Diversités des environnements de } \\
\text { déploiement }\end{array}$ & $\begin{array}{l}\text { Environnement d'intérieur type } \\
\text { laboratoire }\end{array}$ & $\begin{array}{c}\text { Environnements d'intérieur type } \\
\text { laboratoire, d'extérieur (parking, } \\
\text { jardins..) et mobile (smartphones et } \\
\text { véhicules) }\end{array}$ \\
\hline Modularité physique & N/A & N/A \\
\hline $\begin{array}{c}\text { Contrôle et personnalisation du } \\
\text { déploiement }\end{array}$ & Limités & Limités \\
\hline
\end{tabular}

Tableau 1. Comparaison de FIT/IoT-Lab et SmartSantander

Le tableau 1 résume les caractéristiques de deux des plateformes les plus imposantes. Elles ont été retenues en raison de leur taille mais aussi de leur longévité. Ce critère de longévité est lié à la visibilité 
de la plateforme en termes d'évolutions, information fournie par les statistiques Github et des sites web associés à ces testbeds. En dépit des caractéristiques prometteuses répertoriées, force est de constater que, dans la plupart des cas, les plateformes considérées ne proposent pas de mécanisme facilitant la transition d'une couche physique à une autre. Certes, leurs déploiements englobent des sous-réseaux différents en termes de technologies de communication mais évaluer une même solution sur ces deux architectures exige de développer à nouveau l'implémentation. Par ailleurs, il est parfois nécessaire de mener les expérimentations dans un environnement proche de celui envisagé pour le déploiement réel (mines, tunnels...). De ce point de vue, bien que la plateforme la plus active, FIT/IoT-Lab, autorise la sélection de sous-ensembles de nœuds pour constituer la topologie de test, l'environnement demeure homogène (typiquement une pièce dans un laboratoire). Les possibilités de contrôle et de personnalisation, de même que la capacité à approcher le site cible, sont donc limitées.

Notre approche pour le cost-effective testbed permet de lever ces contraintes. En effet, la plateforme proposée dans cet article obéit à une stratégie de minimisation des coûts (utilisation de composants sur étagère, adoption des codes du monde libre...), ce qui permet d'équiper les sites à surveiller moyennant un investissement raisonnable. Néanmoins, cette volonté de raisonner les coûts n'affecte pas la richesse des outils d'interaction proposés et à l'étude dans le projet Ophelia, qui vise à proposer des outils de programmation à distance, de récupération des données et de mise à disposition des résultats d'expérimentation.

En ce qui concerne la modularité physique, la section suivante s'attachera à présenter notre réponse à cette problématique, par la présentation de notre architecture et l'instanciation de cette architecture sur différents exemples.

\section{Présentation de la famille WiNo*}

Comme nous avons pu le voir dans les sections précédentes, il existe un fort besoin de plateforme ouverte permettant le prototypage rapide de protocole réseau, mais aussi de dispositifs connectés, pour les réseaux de capteurs sans fil et le réseau de collecte de l'Internet des objets. L'évaluation réelle et pragmatique constitue une attente forte. Dans ce chapitre, nous présentons un modèle d'architecture présentant des caractéristiques de grande versatilité et répondant à ces besoins.

L'architecture proposée est un modèle de nœud ouvert, "WiNo", contraction de Wireless Node. Cette architecture se veut ouverte et la plus générique possible pour permettre à la fois un prototypage rapide en terme de réseaux et protocoles, mais aussi, de manière globale, le prototypage complet d'objets connectés permettant l'évaluation pragmatique par l'usage protocolaire et applicatif. Cette architecture a été proposée selon les trois principes et objectifs suivants :

- intégration rapide de nouvelles couches physiques : une des difficultés dans le contexte de l'évaluation des protocoles est la nécessité de développer à nouveau les solutions au moment de changer de plateforme physique,

- facteur de forme adapté à l'IoT : dans une perspective de transfert de technologie des laboratoires vers l'industrie, les nœuds communicants sont conçus pour pouvoir s'intégrer tels quels dans la plupart des scénarii, sans nécessiter une phase de miniaturisation,

- capacité à supporter une large palette de capteurs pour des applications variées : la plateforme que nous proposons a pour vocation de devenir un outil pour des chercheurs de domaines différents du nôtre. Les applications et les interactions avec l'environnement ne seront donc pas les mêmes.

L'architecture comprend à la fois des briques matérielles (hardware) et logicielles (software). D'un point de vue matériel, la solution repose sur l'écosystème Arduino, bien connu pour son intérêt et son 
efficacité dans le prototypage rapide de petits systèmes électroniques. Ce choix a été affiné avec la sélection d'un module microcontrôleur de cet écosystème adapté aux besoins : le module retenu, le Teensy 3.x, dispose de plus de mémoire et d'un processeur plus performant que la plupart des modules de l'écosystème Arduino. Cette mémoire est notamment nécessaire pour l'évaluation des performances des protocoles où, en phase d'évaluation, les résultats sont mémorisés pour ne pas consommer de temps CPU. D'un point de vue logiciel, plusieurs briques ont été développées de manière à abstraire l'interface avec le composant émetteur-récepteur (transceiver). Enfin, et en vue d'une évaluation par l'usage, le modèle comprend une batterie de type Lithium Polymère (Li-Po) pour faciliter l'usage du prototype en situation mobile. La figure 1 représente ce modèle d'architecture WiNo. Il a ensuite été décliné dans quatre versions complémentaires, illustrant sa bonne généricité. Les caractéristiques de chacune de ces versions sont résumées dans le tableau 4 ; ces versions sont présentées dans les sections suivantes. Le site [WIN 17] regroupe toutes les informations au sujet des différents nœuds WiNo.

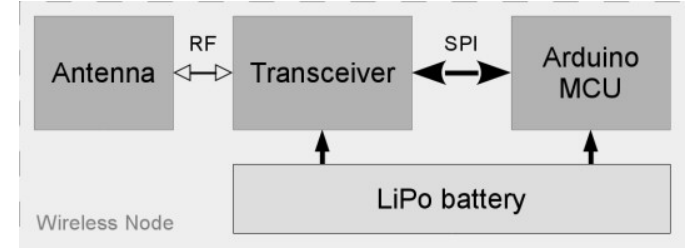

Figure 1. Architecture typique du nœud WiNo

L'objectif avoué de notre contribution est de faire école. Même si dans un premier temps, l'accès à notre plateforme est ouvert à la communauté scientifique, l'intention est de pousser à la mise en place de testbeds partageant certaines caractéristiques mais placés sous des tutelles différentes. En plus de donner une impulsion à une recherche axée prototype, cette stratégie va renforcer la garantie de reproductibilité des résultats. Pour que la modification des sous-systèmes physiques du WiNo soit efficace, nous avons adopté une approche modulaire du logiciel : pour éviter un couplage fort entre les couches basses (liaison de données, réseau) et la couche physique, une librairie permettant de piloter le composant matériel est développée. De cette manière, le développeur peut se focaliser sur la couche correspondant à sa contribution à étudier. Cette stratégie enrichit la phase d'évaluation en rajoutant un degré de liberté aux expérimentations et en permettant de choisir la couche physique la plus adaptée à la suite protocolaire.

Les sections suivantes illustrent plusieurs instanciations de notre modèle.

\subsection{WiNoRF22 et TeensyWiNo}

Le WiNoRF22 (figure 2) est la première instanciation de l'architecture proposée, tandis que le TeensyWiNo, fabriqué et distribué par Snootlab.com, est une version industrialisée de l'architecture WiNo, comprenant de nombreux capteurs supplémentaires.

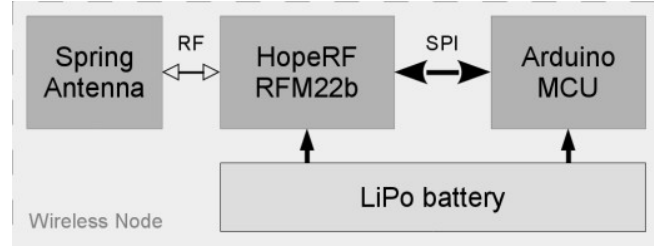

Figure 2. Architecture du nœud WiNoRF22

Les deux nœuds (figure 3) partagent les mêmes caractéristiques en termes de couche physique. La radio qui équipe les nœuds est un transceiver HopeRF RFM22b; bien que cette radio ne soit pas compatible avec les transceivers IEEE 802.15.4 classiques, cette radio "Sub-GHz» présente des caractéristiques similaires en terme de format de trame, mais avec un large choix de configurations possibles sur les modulations (OOK, FSK, GFSK), la fréquence de travail (200 à $900 \mathrm{MHz}$ ), le débit (1 
à $125 \mathrm{kbps}$ ) et la largeur de bande (1 à $125 \mathrm{kHz})$. Ce large panel permet au WiNoRF22 et au TeensyWiNo de s'adapter à de nombreux usages et besoins sur le plan protocolaire (bas débit sur une topologie en étoile, sur une portée de 300 mètres, ou, débit plus élevé, pour élaborer des protocoles mesh avec une portée plus réduite par exemple).

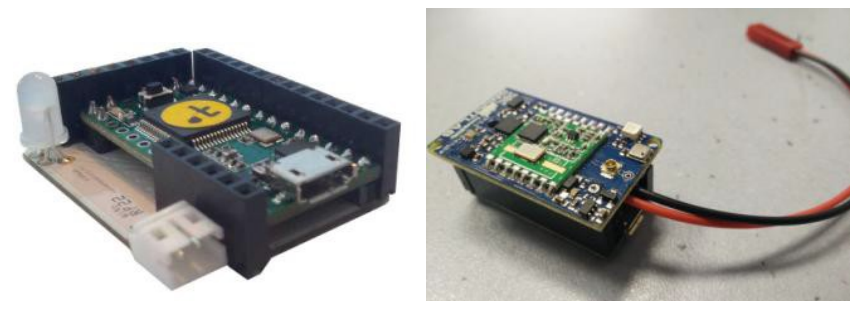

Figure 3. WiNo (gauche) et TeensyWiNo (droite)

IMPORTANT. Dans le domaine des prototypes pour les réseaux de capteurs et les objets connectés, un point très important concerne la maîtrise de la dépense énergétique liée au nœud et aux protocoles de communication. De manière générale, si les objets connectés présentent des autonomies inférieures à leurs équivalents «non-connectés », leur acceptation par les utilisateurs est difficile. Le design du WiNo tient compte de cette exigence et a été évalué en situation d'usage. Le tableau 2 indique l'autonomie énergétique d'un TeensyWiNo mesurée en situation d'usage, en fonction de la capacité de la batterie LiPo connectée, pour deux scénarios : capteur de température (1 mesure toutes les 10 secondes) ou capteur de mouvement par l'accéléromètre (un mesure deux fois par seconde). De manière plus générale, les principaux postes de consommation énergétique du WiNo sont indiqués dans le tableau 3.

\begin{tabular}{|c|c|c|}
\hline & Batterie 850 mA.h & Batterie $1600 \mathrm{~mA} . \mathrm{h}$ \\
\hline Capteur de température & 50 jours & 117 jours \\
\hline Capteur de mouvement & 45 jours & 101 jours \\
\hline
\end{tabular}

Tableau 2. Autonomie énergétique d'un TeensyWiNo en situation d'usage

\begin{tabular}{|c|c|c|}
\hline Postes de consommation & Etat & Puissance consommée $(\mathrm{Vcc}=3.7 \mathrm{~V})$ \\
\hline \multirow{7}{*}{$\begin{array}{c}\text { CPU } \\
\text { Freescale MK20DX256 VLH7 }\end{array}$} & Actif, $96 \mathrm{MHz}$ & $129 \mathrm{~mW}$ \\
\hline & Actif, $72 \mathrm{MHz}$ & $103 \mathrm{~mW}$ \\
\hline & Actif, $48 \mathrm{MHz}$ & $88.8 \mathrm{~mW}$ \\
\hline & Actif, $24 \mathrm{MHz}$ & $55.5 \mathrm{~mW}$ \\
\hline & Sleep, réveil par LPTMR & $2 \mathrm{~mW}$ \\
\hline & Deepsleep, réveil par LPTMR & $650 \mu \mathrm{W}$ \\
\hline & Hibernate, réveil par LPTMR & $<30 \mu \mathrm{W}$ \\
\hline \multirow{4}{*}{ Transceiver HopeRF RFM22b } & Transmit (10 dBm) & $76 \mathrm{~mW}$ \\
\hline & Receive & $57 \mathrm{~mW}$ \\
\hline & Idle & $26 \mathrm{~mW}$ \\
\hline & Sleep & $<5 \mu \mathrm{W}$ \\
\hline
\end{tabular}

Tableau 3. Consommation énergétique du TeensyWiNo

Par rapport au WiNoRF22, le TeensyWiNo (figure 4) comprend de nombreux capteurs, permettant le prototypage de nombreux objets communicants sans ajout d'électronique supplémentaire, comme nous le verrons dans la section suivante. 


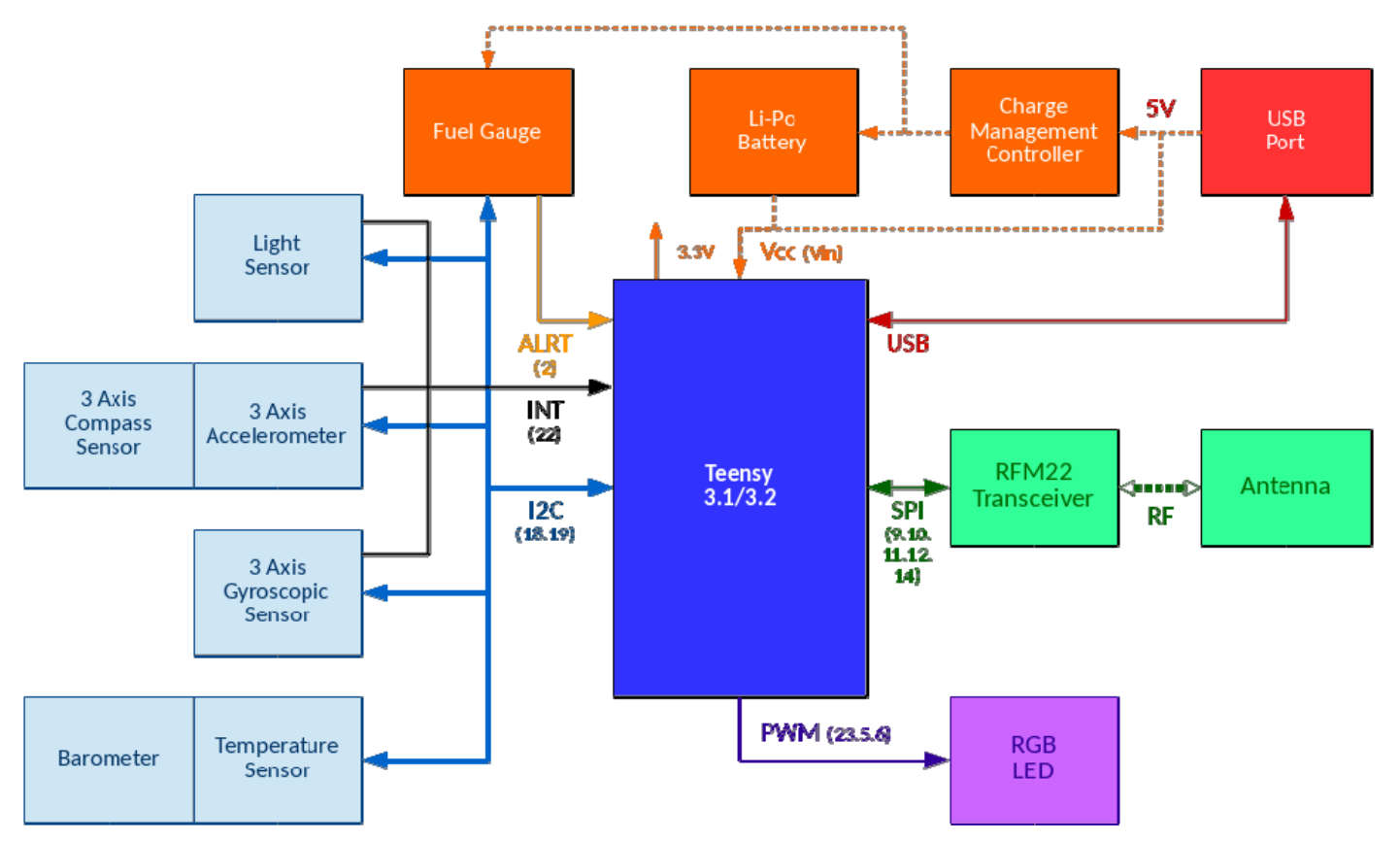

Figure 4. Architecture du nœud TeensyWiNo

\subsection{WiNoLoRa}

Le WiNoLoRa est une déclinaison du WiNo exploitant une couche physique LoRa (Long-Range). LoRa [BOR 16] et un mode de transmission en bande étroite très bas débit qui permet d'atteindre des portées très élevées comparées aux modes de transmission classiquement utilisés dans les réseaux de capteurs sans fil ; des portées de plusieurs centaines de mètres, voire de plusieurs kilomètres, sont atteignables avec des puissances d'émission de l'ordre de la dizaine de milliwatts.

Le WiNoLoRa reprend le principe du WiNo en remplaçant le transceiver par un Hope RFM95 compatible LoRa dans la bande des $868 \mathrm{MHz}$. Le prototype matériel que nous avons réalisé est présenté à la figure 6 , il est basé sur l'architecture détaillée en figure 5 .

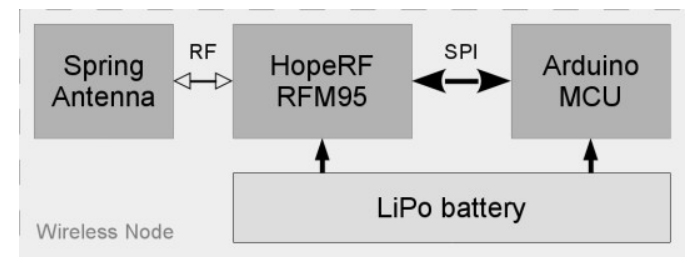

Figure 5. Architecture du nœud WiNoLoRa

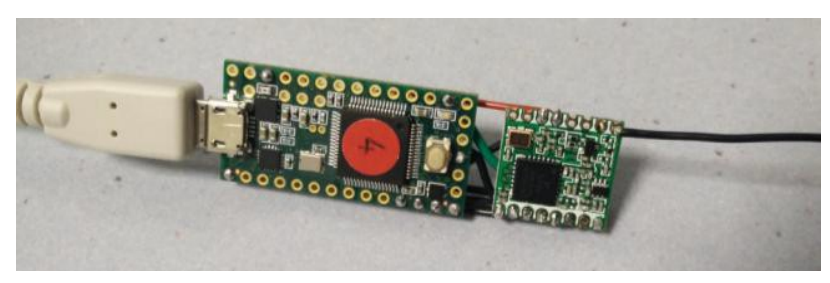

Figure 6. Prototypage du nœud WiNoLoRa

Le WiNoLoRa permet aussi bien de prototyper des protocoles de niveau 2 et plus, sur une couche PHY LoRa, que de se connecter à des réseaux LoRaWAN existants, à condition d'utiliser une implémentation de l'empilement protocolaire LoRaWAN, dont plusieurs versions ouvertes sont disponibles [IOT 17]. 


\subsection{DecaWiNo}

Le DecaWiNo est une déclinaison du WiNo qui rend accessibles les spécificités de la couche physique Ultra-Wide Band (UWB). En plus de la fonction de communication, cette PHY permet la mise en œuvre de protocoles de ranging ou mesure de distance, basés sur le temps de propagation du signal radio. Cette variante du WiNo (figure 7) exploite l'un des quelques émetteurs-récepteurs UWB disponibles sur le marché, le DW1000 de DecaWave. Ce module a l'avantage de respecter dans une large mesure le standard IEEE 802.15.4-2007 décrivant le fonctionnement d'une couche physique UWB.

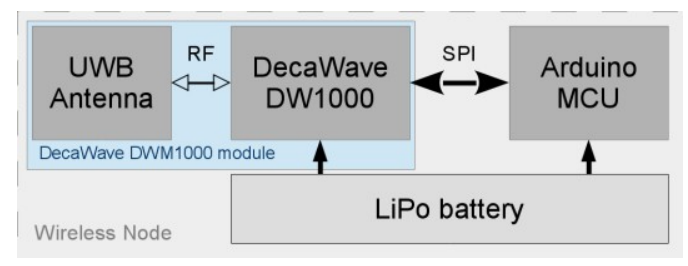

Figure 7. Architecture du nœud DecaWiNo

Le pilotage de ce transceiver se fait à travers la brique logicielle DecaDuino [VAN 16]. Cette dernière a été développée par notre équipe et permet l'accès aux fonctionnalités de communication mais aussi aux estampilles temporelles générées lors des transmissions et réceptions de trames. Notre bibliothèque fournit également une estimation de la différence entre les horloges des nœuds communicants et la possibilité de préprogrammer l'instant d'émission d'un bit spécifique des trames, dit RMARKER. Cette transmission retardée permet d'économiser des messages puisque l'instant d'émission prévu peut être porté par la même trame [FOF 16] (figure 8), tandis que la capacité à caractériser les horloges d'émission et de réception nous a permis de corriger dynamiquement les estimations de temps de propagation [DAL 15].
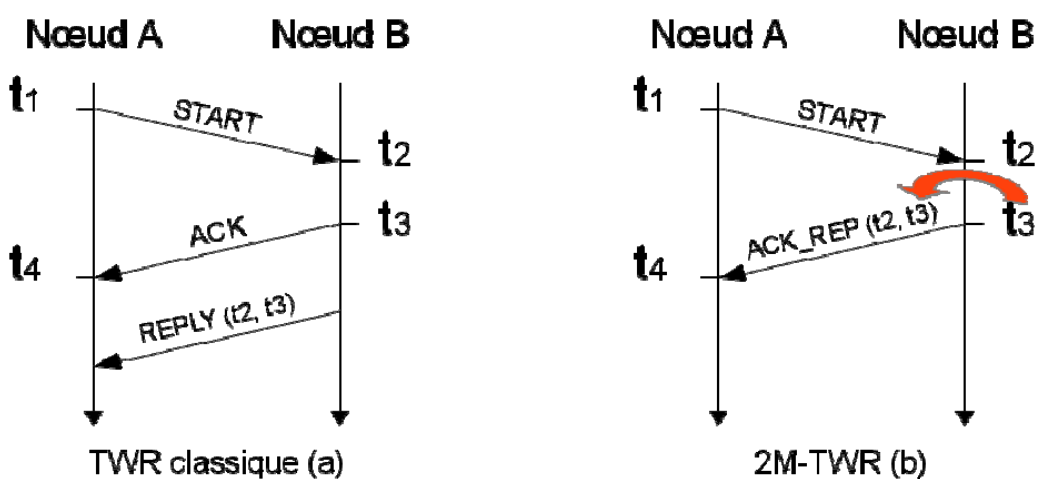

Figure 8. Diagrammes de séquence des protocoles TWR et 2M-TWR

DecaDuino offre des points d'entrée pour le développement de nouveaux protocoles sous la forme d'implémentations de protocoles connus comme le Two-Way Ranging (TWR) et le Symmetric DoubleSided Two-Way Ranging (SDS-TWR). Ces versions accèdent directement à la couche physique par des Service Access Points (SAP) dédiés (figure 9), ce qui évite aux protocoles de ranging de subir les effets du protocole d'accès au médium choisi. 

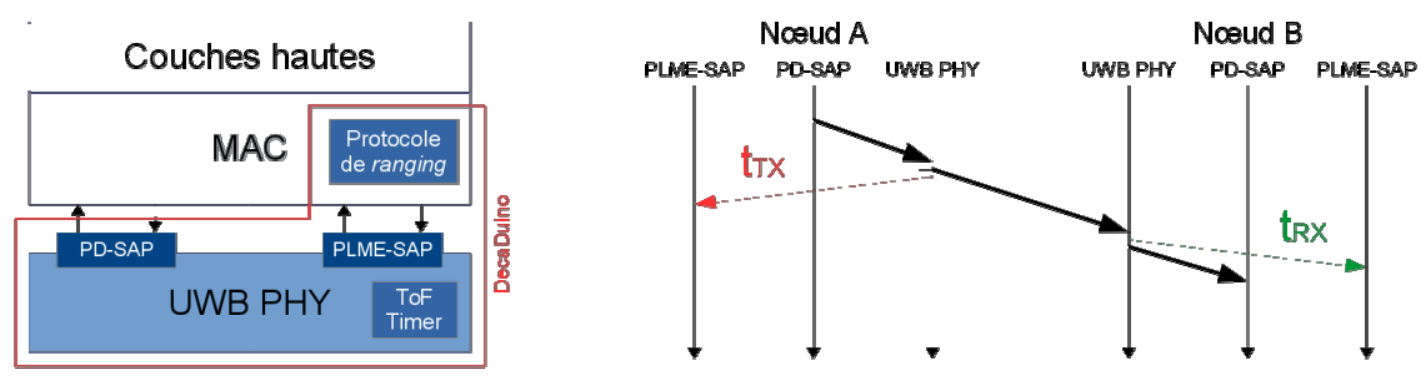

Figure 9. DecaDuino dans l'empilement protocolaire

\subsection{Synthèse des nœuds WiNo}

L'architecture WiNo propose donc un modèle de nœud ouvert et se veut la plus générique possible pour permettre le prototypage rapide en réseaux et protocoles, mais aussi, de manière plus globale, le prototypage complet d'objets connectés grâce à l'intégration simple rapide de nouvelles couches physiques, avec un facteur de forme adapté à l'évaluation par l'usage et avec une capacité à supporter une large palette de capteurs pour des applications variées. Le tableau 4 résume les quatre déclinaisons présentées.

\begin{tabular}{|c|c|c|c|c|}
\hline & TeensyWiNo & WiNoRF22 & WiNoLoRa & DecaWiNo \\
\hline Usage & \multicolumn{2}{|c|}{ IoT et RCSF } & Long-range & Short-range, loca \\
\hline CPU/RAM & \multicolumn{4}{|c|}{$\begin{array}{l}\text { PJRC Teensy 3.2, compatible Arduino (Freescale MK20DX256VLH7) ARM Cortex M4 72MHz, 64kB } \\
\text { RAM, 256kB Flash }\end{array}$} \\
\hline $\begin{array}{l}\text { Modulation } \\
\text { Transceiver }\end{array}$ & \multicolumn{2}{|c|}{ OOK/FSK/GFSK HopeRF RFM22b } & $\begin{array}{l}\text { LoRa/OOK/FSK/GFSK } \\
\text { HopeRF RFM95 }\end{array}$ & $\begin{array}{l}\text { UWB DecaWave } \\
\text { DW1000 }\end{array}$ \\
\hline Librairie & \multicolumn{3}{|c|}{ RadioHead } & DecaDuino \\
\hline Capteurs & $\begin{array}{l}\text { Temp, Lum, Baro, } \\
\text { Accéléro, Magnéto, Gyro }\end{array}$ & \multicolumn{3}{|c|}{ Température, luminosité } \\
\hline Autres & \multicolumn{4}{|c|}{ RGB LED, GPIOs, PWM, ADC/DAC, SPI, I2C, CANbus } \\
\hline Disponibilité & snootlab.com & & DIY & \\
\hline
\end{tabular}

Tableau 4. Variantes de WiNo

Plusieurs résultats et exemples d'utilisation de l'architecture WiNo sont détaillés dans la section suivante.

\section{Résultats et exemples d'utilisation}

Notre plateforme WiNo a été utilisée à de nombreuses reprises pour des applications variées, suivant les versions de nœuds WiNo. Nous présentons dans cette section quelques exemples représentatifs d'utilisation pour respectivement les nœuds WiNo à $433 \mathrm{MHz}$ (WiNoRF22 et TeensyWiNo), les WiNo équipés d'un tranceiver permettant de travailler en LoRa (WiNoLoRa), et les WiNo en version UWB (DecaWiNo). 


\subsection{WiNo et TeensyWiNo}

\subsubsection{Les nœuds WiNoIR [BOS 16]}

Un habitat classique héberge de nombreux équipements pilotables par télécommandes infrarouges (IR), comme par exemple les téléviseurs, lecteurs de DVD, vidéoprojecteurs, systèmes home-cinémas pour les plus classiques, mais aussi lits médicalisés, hottes aspirantes, robots aspirateurs autonomes, WC japonais, climatisations, pour les habitats de plus haute technologie... Ces différents équipements peuvent former un IoT local moyennant la mise en œuvre de passerelles entre le système de contrôle infrarouge et le réseau local de l'habitat intelligent. Cet IoT peut contribuer au confort de l'habitant mais peut aussi devenir un tremplin vers l'autonomie pour une personne à mobilité réduite. En ce sens, quelques solutions isolées existent; elles reposent souvent sur l'utilisation du Smartphone comme élément de pilotage et de contrôle. Une première catégorie correspond aux IR-Blasters : dans leur version de base, ils commandent une diode IR émettrice connectée sur le port audio jack du Smartphone à l'aide d'une application adaptée. Quelques Smartphones modernes intègrent maintenant cette diode émettrice.

Une seconde catégorie fait appel à des passerelles dédiées, comme par exemple le produit Broadlink [BRO 17]. Dans cette configuration, le Smartphone communique avec la passerelle et l'intelligence du système, généralement propriétaire, est contenue dans la passerelle. Une base de données des codes IR les plus classique est utilisée et il est souvent compliqué voire impossible de piloter des équipements non traditionnels ou de grande distribution. L'inconvénient principal de cette classe de solutions est la quasi-obligation d'utiliser un Smartphone pour piloter les équipements de la maison.

D'autres solutions spécifiques existent, dédiées aux personnes handicapées comme les téléthèses d'environnements appelés aussi contrôles d'environnement ; ces systèmes permettent à une personne en situation de handicap moteur d'interagir à distance avec son environnement. Ces dispositifs sont pourvus de techniques d'interaction homme-machine adaptées aux situations de handicap des personnes, que ceux-ci soient dues aux déficiences ou à l'environnement (commande de l'objet distant) comme le système MATT [VEL 15], le système Pictocom [BOC 16], [ACC 17] ou encore le système House Mate Control [DOM 17], un contrôle d'environnement via un téléphone ou une tablette sous Android par infrarouge et radio via la D-Box et la domotique sans fil. Enfin, il ne faut pas oublier les classiques et bien connus systèmes de déport de télécommandes IR, qui sont de simples répéteurs, généralement par une couche radio $433 \mathrm{MHz}$, pour piloter depuis une pièce via sa télécommande d'origine, un équipement situé dans une autre pièce, donc hors de portée IR à cause de la distance ou des obstacles tels que les murs. Ces équipements ne sont pas prévus pour être interfacés avec un autre réseau.

Afin de répondre à ce besoin, nous avons conçu des nœuds WiNoIR (figure 10), assurant le pilotage par IR d'équipements domotiques, et connectés par un réseau radio de l'habitat aux autres équipements et réseaux existants classiques. Ces WiNoIR s'intègrent dans un système de commande des équipements ouvert et évolutif. Ce dispositif a pour ambition de limiter la prolifération des télécommandes IR sans pour autant inhiber leur fonctionnement. Le pilotage est possible à partir de n'importe quelle source locale connectée au réseau de la maison : ordinateur, tablette, Smartphone, capteur domotique, organe de contrôle et de décision..., mais aussi à partir d'un hôte distant via Internet. 


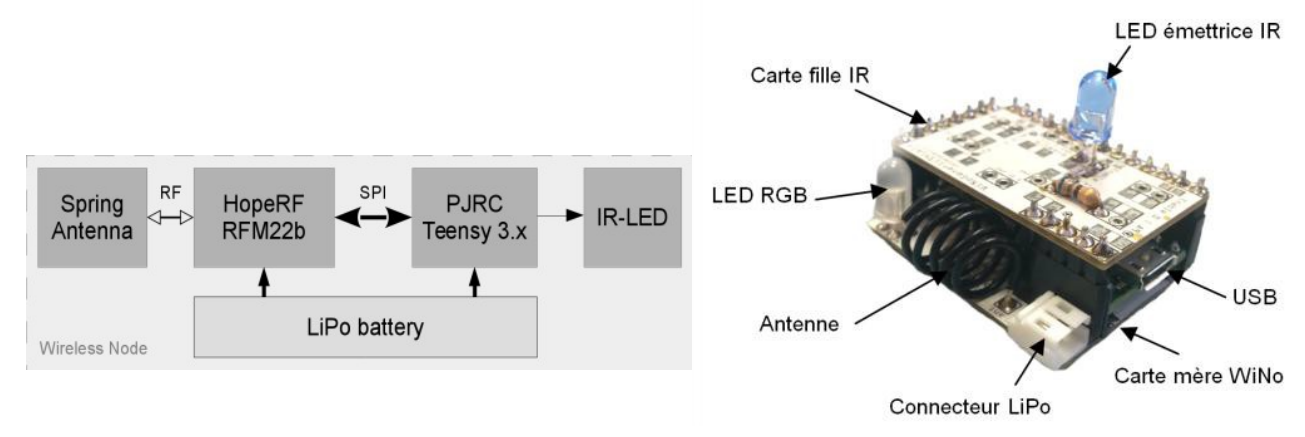

Figure 10. Le nœud WiNolR

Les nœuds WiNo étant basés sur une architecture matérielle Teensy [TEE 17], nous nous sommes naturellement orientés vers l'écosystème Arduino et avons utilisé la librairie IrRemote développée initialement par Ken Shirriff [IRR 17]. Cette librairie, développée pour les cartes Arduino UNO, a été adaptée pour fonctionner également sur les modules Teensy. Elle offre la possibilité d'apprendre les codes des télécommandes existantes (avec un nœud spécifique équipé d'une LED réceptrice), et de rejouer ces codes par la suite. La première étape est d'apprendre les codes IR des télécommandes des équipements que l'on veut piloter. La librairie IrRemote permet de recevoir les codes IR d'un récepteur connecté sur un port d'entrée du Teensy. Ces codes sont alors traités, reconnus et identifiés parmi les codes les plus standards (RC5, RC6, Sony, Samsumg...) et mémorisés. Une autre fonction permet de rejouer ces codes via une diode d'émission IR connectée sur un port de sortie. La librairie IrRemote est suffisamment générique pour s'adapter aux modulations les plus standards $(38 \mathrm{KHz})$, mais il est également possible de modifier ce paramètre. Pour les codes IR non reconnus, il est également possible de travailler en mode RAW. Ce mode, plus gourmand en mémoire, code en fait chaque ordre de télécommande par une succession d'impulsions IR, dont la durée est mémorisée en millisecondes, ainsi que la durée entre deux flashs IR successifs. C'est ce mode que nous avons privilégié, car il fonctionne aussi bien pour les télécommandes connues que pour celles utilisant des codages IR propriétaires.

Les nœuds WiNoIR sont actuellement utilisés dans la maison intelligente de l'IUT de Blagnac [MIB 17], en particulier lors de tests réels de l'utilisation de la maison par des personnes âgées dans le projet de recherche SENUM (Séniors et Numérique) en partenariat avec AG2R LA MONDIALE, et d'autres travaux de recherche [BOU 16], [VDB 16]. Ils nous permettent, à distance (au sein du même réseau local, ou potentiellement depuis une machine distante reliée au réseau Internet) de piloter tous les équipements de la maison, même ceux pour lesquels aucune interface IP n'est prévue. La méthodologie utilisée pour leur conception, et en particulier l'apprentissage des codes IR, les rend interopérables avec n'importe quel équipement disposant nativement d'une télécommande IR classique.

\subsubsection{Exemple d'objets d'interaction : la canne}

En modifiant les capteurs et actionneurs de nos WiNo, nous avons conçu des objets connectés d'interaction : canne connectée et cube d'interaction. L'objectif général est de faciliter la communication entre le domicile et l'habitant, en particulier dans le cas du maintien à domicile des personnes âgées. Dans cette section, nous présenterons notre canne de marche connectée.

Afin d'assurer le suivi des personnes âgées, il est courant de les équiper de médaillons ou autres bracelets. Ces équipements peuvent être utilisés pour détecter les chutes, mesurer l'activité, la marche... Néanmoins, ces équipements sont assez contraignants, et il est courant que les personnes âgées, après les avoir posés avant une douche, oublient de les remettre. Partant du constat que la canne de marche est un équipement couramment utilisé par ces personnes, nous avons mis en place dès 2012 le projet CANet, dont l'objectif est de suivre le comportement d'une personne via sa canne de marche instrumentée. 


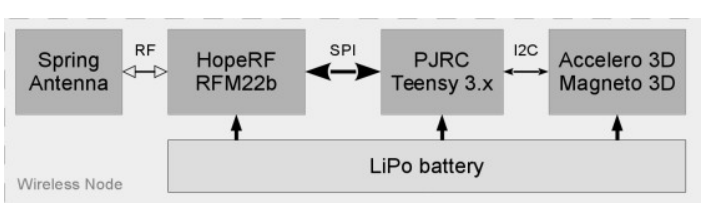

Figure 11. Architecture du nœud WiNo spécifique au projet CANet

La canne est équipée d'un nœud WiNo spécifique (figures 11 et 12) assez étroit pour se loger dans le tube même de la canne, possédant un accéléromètre 3D, un magnétomètre $3 \mathrm{D}$, un module Teensy, un tranceiver radio RFM22b, et une batterie LiPo. La canne, après traitement local, est capable d'évaluer le nombre de pas effectués, d'en déduire la distance parcourue, de détecter les chutes suivies d'une inactivité et transmettre par radio des alertes [LAC 16]. Une version CANet LoRaWAN est également à l'étude et permettrait également d'accompagner la personne en extérieur, tout en assurant sa localisation sans recours à un GPS.
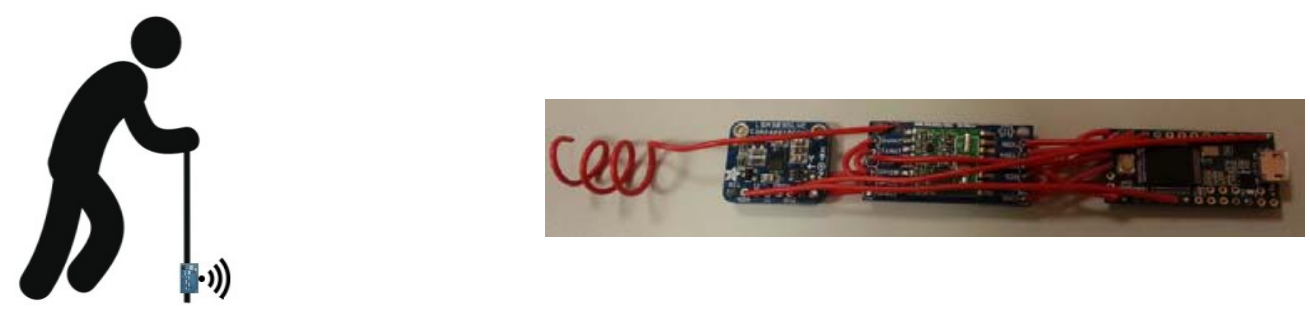

Figure 12. La canne connectée et son nœud WiNo dédié

\subsection{WiNoLoRa}

L'un des intérêts de l'architecture WiNo est la possibilité d'élaborer et tester des protocoles de niveau 2 et plus, sur les couches physiques proposées par le transceiver associé. Afin de permettre cette élaboration, il convient de bien connaître la couche physique. Dans cette section, nous présentons une étude des performances de la couche PHY LoRa, en termes de débit et temps sur le médium. Cette étude a été réalisée à l'aide du prototype WiNoLoRa.

REMARQUE. LoRa est une technologie qui est systématiquement présentée comme un mode de transmission « bas-débit »... Mais quel est l'ordre de grandeur ? Cette étude, réalisée à partir du nœud WiNoLoRa, permet d'obtenir des ordres de grandeur en termes de débit et d'occupation temporelle du médium.

Afin de réaliser cette expérimentation, deux nœuds WiNoLoRa ont été configurés, le premier en transmetteur, et le second en récepteur. Le scénario est le suivant : le transmetteur forme des trames d'une charge utile de longueur aléatoire, et le récepteur veille à la bonne réception de ces messages. Le temps sur le canal est mesuré de part et d'autre, et vérifié égal. Le scénario est reproduit pour trois configurations différentes de la PHY LoRa, jouant sur les paramètres Bandwidth (BW), Coding Rate (CR) et Spreading Factor (SF) :

- $\mathrm{BW}=125 \mathrm{kHz}, \mathrm{CR}=4 / 5, \mathrm{SF}=7$ (128 bits de code d'étalement),

- $\mathrm{BW}=125 \mathrm{kHz}, \mathrm{CR}=4 / 8, \mathrm{SF}=12$ (4096 bits),

- $\mathrm{BW}=31.25 \mathrm{kHz}, \mathrm{CR}=4 / 8, \mathrm{SF}=9$ (512 bits). 

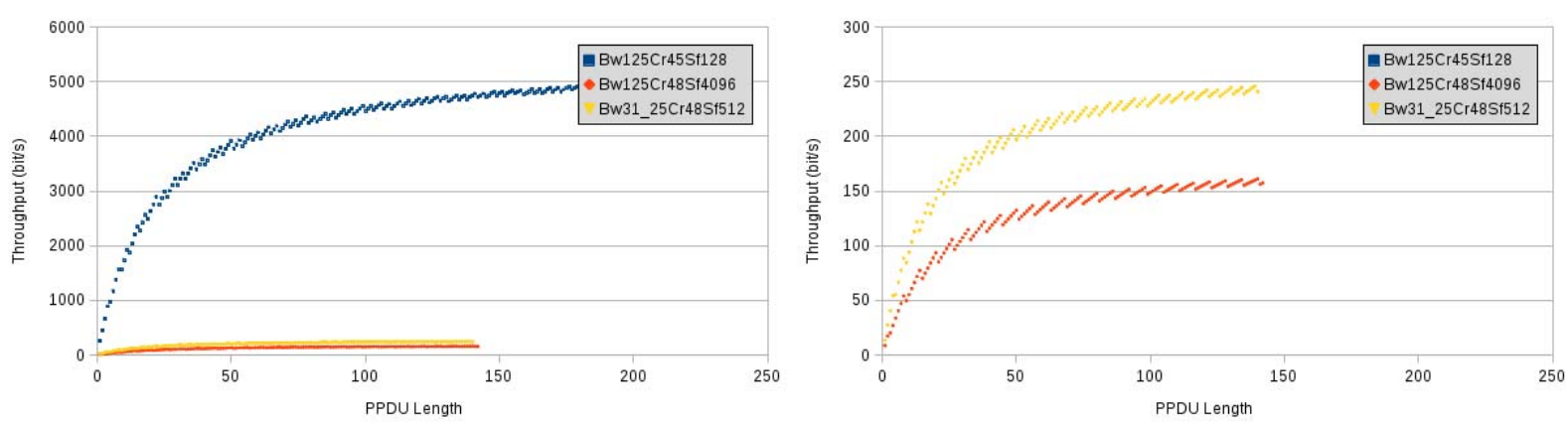

Figure 13. Débits d'un lien utilisant une couche PHY LoRa en fonction des paramètres PHY

Les résultats (figure 13) montrent que, conformément à ce qui est attendu, le débit augmente avec la longueur de la charge utile (PPDU), ce qui s'explique par la présence d'une charge protocolaire native (en-tête, $\mathrm{CRC}$ ) du protocole de niveau physique ; cette charge est « rentabilisée » sur les messages de longueur importante. Alors que le premier scénario permet d'atteindre un débit efficace de près de $5 \mathrm{kbit} / \mathrm{s}$, les deux autres paramétrages permettent d'atteindre respectivement des débits de 150 et $250 \mathrm{bit} / \mathrm{s}$ pour des charges utiles de 150 octets. Il est à noter que ces débits sont à considérer en tenant compte du temps sur le canal, qui peut atteindre plus de 7 secondes dans le scénario 2, pour une charge utile de 150 octets (figure 14). En effet, la réglementation impose un taux d'utilisation (duty-cycle) limité par unité de temps.

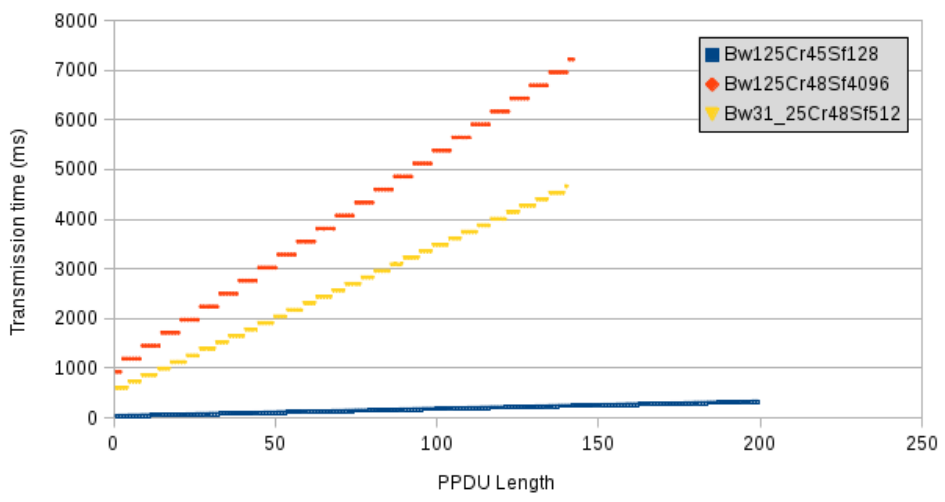

Figure 14. Temps sur le médium en fonction des paramètres $P H Y$

Nos travaux actuels portent en particulier sur l'utilisation conjointe de plusieurs technologies complémentaires dédiées à l'IoT, dont LoRa [GON 16].

\subsection{DecaWiNo}

La librairie DecaDuino s'accompagne d'implémentations de protocoles connus comme TWR et SDS-TWR. Ces deux protocoles de ranging sont souvent comparés en termes de robustesse par rapport à la qualité des horloges, plus précisément en termes de skew. Le protocole SDS-TWR a notamment été mis en avant dans [IEE 07] pour sa capacité à mitiger les effets délétères de la différence entre les horloges de l'émetteur et du récepteur sur les estimations de temps de propagation. Malheureusement, SDS-TWR exige un nombre de trames transmises important, comparativement à TWR.

À partir de la fonctionnalité de transmission retardée fournie par notre bibliothèque, nous avons proposé le protocole 2M-TWR de ranging plus économe en termes d'overhead.

La figure 15 représente l'erreur de ranging obtenue avec $2 \mathrm{M}$-TWR comparée à la version classique de TWR dans une situation LOS (ligne de vue). Comme on peut le voir, l'erreur moyenne reste dans les 
$20 \mathrm{~cm}$; cependant, la fonctionnalité de transmission retardée entraîne des écarts importants entre les mesures, que nous sommes actuellement en train d'étudier et compenser par un protocole spécifique.

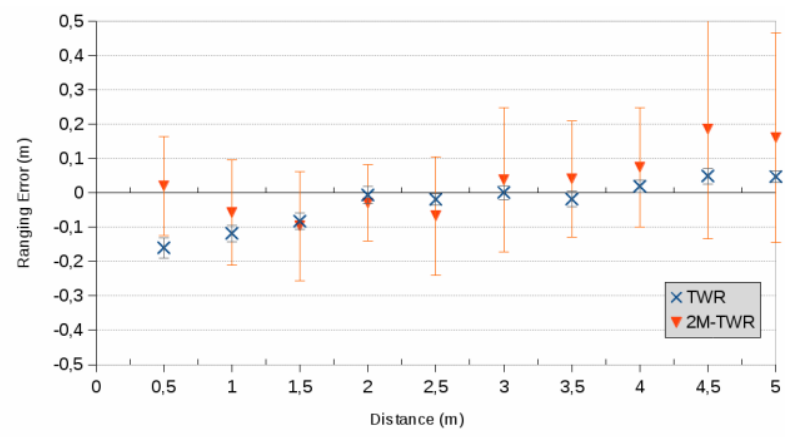

Figure 15. Performances du protocole 2M-TWR comparé à la version classique de TWR

\subsection{Synthèse et analyse comparative}

L'un des moteurs du développement des plateformes de prototypage présentées dans cet article est la réconciliation entre l'étude de performances théoriques et la réalité du terrain. En vue de s'intégrer à différents projets et approches, le testbed, en plus de disposer d'une interface de commande riche, se doit d'être flexible et modulaire : le coût du basculement d'une couche physique à l'autre doit être raisonnable.

La famille de nœuds WiNo répond à cette problématique en présentant une interface standardisée à la couche Liaison de Données. Les équipes de recherche désireuses de mener des expérimentations en environnement réel n'ont plus qu'à faire leur choix en exploitant les indications du tableau 5.

\begin{tabular}{|c|c|c|c|}
\hline & Teensy WiNo/WiNoRF22 & WiNoLoRa & DecaWiNo \\
\hline Portée radio & $\begin{array}{c}100 \mathrm{~m} \text { en intérieur (modulation } \\
\text { GFSK) }\end{array}$ & $30 \mathrm{~km}$ en ligne de vue & $20 \mathrm{~m}$ en ligne de vue \\
\hline Débits supportés & 0,123 à $256 \mathrm{kbit} / \mathrm{s}$ & 0,3 à $20 \mathrm{kbit} / \mathrm{s}$ & $110,850 \mathrm{et} 6800 \mathrm{kbit} / \mathrm{s}$ \\
\hline Bande de fréquence & $\begin{array}{c}433 / 470 / 868 / 915 \mathrm{MHz} \\
\text { (ISM) }\end{array}$ & $868 \mathrm{MHz}$ & $3,5 \mathrm{GHz}$ à $6.5 \mathrm{GHz}$ \\
\hline Ressources disponibles & API : réception / transmission de messages radio ; configuration de l'émetteur-récepteur \\
\hline Applications & $\begin{array}{c}\text { Monitoring à distance Réseaux } \\
\text { multi-sauts à grande échelle }\end{array}$ & $\begin{array}{c}\text { Réseaux mono-sauts à grande } \\
\text { échelle }\end{array}$ & $\begin{array}{c}\text { Mesure de distance par temps de } \\
\text { propagation }\end{array}$ \\
\hline
\end{tabular}

Tableau 5. Caractéristiques techniques et recommandations applicatives

\section{Conclusion et perspectives}

L'internet des objets, terme à la mode s'il en est, recouvre divers aspects de la collaboration entre des réseaux différents en termes de services, de taille, mais aussi de technologies de communication. Cette hétérogénéité est un caractère assumé mais il introduit des exigences au niveau de l'étude du réseau de collecte de l'Internet des Objets connectés, appelé de plus en plus souvent maintenant DLIoT pour Device-Layer-IoT. En effet, puisque diverses couches physiques existent, des moyens doivent être mis en œuvre pour étudier leur adéquation, en termes de capacités intrinsèques mais aussi en 
termes de cohésion avec les couches supérieures, et d'interopérabilités entre elles. Les travaux présentés dans cet article ont pour objectif de répondre à ce besoin: la plateforme matérielle WiNo a été conçue dans le respect des principes de l'open source, tant sur le matériel que sur le logiciel. Ouverte et générique, elle accélère la prise en charge de couches physiques innovantes par une couche d'abstraction. Si ce modèle a été adopté et est mis à contribution dans des projets de recherche transversaux, l'objectif à long terme est de faire école et donc de porter divers laboratoires à créer leur propre testbed à des prix abordables. Pour atteindre cet objectif, il nous reste encore quelques étapes à franchir, comme offrir diverses couches MAC en plus de celles actuellement disponibles (CSMA/CA, TDMA...), proposer un middleware et des applications pour grapher les résultats afin d'analyser finement les performances obtenues, et rendre accessible à distance notre testbed. Ces étapes sont en cours de réalisation dans le projet Ophelia soutenu par la région Occitanie. Notre objectif est également d'utiliser ce testbed dans des actions pédagogiques, comme un SPOC associé au MOOC [MOO 17] sur les objets connectés dans lequel nous sommes impliqués, en particulier sur la programmation de protocoles dédiés aux objets connectés sur l'IoT.

\section{Remerciements}

Les auteurs souhaitent remercier Snootlab.com, partenaire du projet Ophelia, et la Région MidiPyrénées/Occitanie pour le cofinancement d'une partie des travaux présentés dans cet article.

\section{Bibliographie}

[ABD 17] AbDelgawad A., Yelamarthi K., «Internet of Things (IoT) Platform for Structure Health Monitoring », Wireless Communications and Mobile Computing, Volume 1, 2017, Article ID 6560797, 10 pages.

[ACC 17] http://www.access-man.com/produits/pictocom

[BOS 16] Van den Bossche A., Blanc Machado M., Val T., Vella F., VigourouX N., «Utilisation des noeuds WiNoIR pour connecter tous les équipements domotiques d'un habitat intelligent », Journée Nationale de l'Internet des Objets - Nouveaux défis de l'Internet des Objets: Interaction Homme-Machine et Facteurs Humains, Paris-Saclay, 29/11/2016.

[BRO 17] http://www.broadlink.com.cn/en/home-en.html

[BOC 16] BoCCA M.-L.. « Evaluation et optimisation d'une aide à la communication chez des patients en situation de handicap (handicap et aide à la communication) », Conférence handicap 2016 , Juin 2016, Paris.

[BOR 16] BOR, M., VIDLER, J. E., ROEDIG, U, « LoRa for the Internet of Things », EWSN '16 Proceedings of the 2016 International Conference on Embedded Wireless Systems and Networks, Canada: Junction Publishing, p. 361-366. $15 / 02 / 2016$

[BOU 16] Bougeois E., Duchier J., Vella F., Blanc Machado M., Van den Bossche A., Val T., Vigouroux N., CAMPO E., "Post-test perceptions of digital tools by the elderly in an ambient environment », International Conference On Smart homes and health Telematics (ICOST 2016), Wuhan, China, 25-27/05/16.

[CAO 08] Cao Q., Abdelzaher T., Stankovic J, He T., « The LiteOS Operating System: Towards Unix Like Abstraction for Wireless Sensor Networks ", Proceedings of the 7th International Conference on Information Processing in Sensor Networks (IPSN 2008), 2008.

[DAL 15] DALCE R., VAN DEN Bossche A., VAL T., " Reducing localisation overhead: a ranging protocol and an enhanced algorithm for UWB-based WSNs », IEEE Vehicular Technology Conference (VTC 2015), Glasgow, Scotland, 11/05/2015-14/05/2015.

[DOM 17] http://www.domodep.com/produits/controle-environnement.html/housematecontrol.html

[DUC 15] Duchier J., Vigouroux N., Bougeois E., Vella F., Boudet B., Rumeau P., « Preliminary study of human interactions during entertainment by a facilitator using a cognitive stimulation medium (Mémoire-RED) in a nursinghome setting», Non-pharmacological Therapies in Dementia, Nova Science Publishers, Vol. 3 N. 2, p. 181-186, 2015. 
[DUN 04] Dunkels A., Gronvall B., Voigt T., " Contiki a Lightweight and Flexible Operating System for Tiny Networked Sensors ", Proceedings of the 9th Annual IEEE International Conference on Local Computer Networks, Washington, DC, USA, 2004.

[FLE 15] Fleury E., Mitton N., Noel T., Adjih C., « FIT IoT-LAB: The Largest IoT Open Experimental Testbed », ERCIM News, ERCIM, 2015.

[FOF 16] Fofana N. I., VAn Den Bossche A., Dalce R., Val T., " An Original Correction Method for Indoor Ultra Wide Band Ranging-based Localisation System », International Conference on Ad Hoc Networks and Wireless (AdHoc-Now 2016), Lille (France), Springer International Publishing, 04-06/07/2016.

[GON 16] Gonzalez N., Van Den Bossche A., Val T.. « Hybrid Wireless Protocols for the Internet Of Things ». IFIP/IEEE International Conference on Performance Evaluation and Modeling in Wired and Wireless Networks (PEMWN 2016), Paris, France, 22-24/11/2016.

[IEE 07] IEEE COMPUTER SocIETY, « Specific requirements Part 15.4: Wireless Medium Access Control (MAC) and Physical Layer (PHY) Specifications for Low-Rate Wireless Personal Area Networks (WPANs) Amendment 1: Add Alternate PHYs », IEEE Standard for Information technology Telecommunications and information exchange between systems, 2007.

[IOT 17] https://iot.iut-blagnac.fr/

[IRR 17] http://github.com/z3t0/Arduino-IRremote

[JAE 14] JAEWOO K., JaIYONG L., JAeHO K., JAESEOK Y., « M2M Service Platforms: Survey, Issues, and Enabling Technologies », IEEE Communications Surveys \& Tutorials, 01/2014, pp 61-76.

[KeT 14] Keranidis S., Giatsios D., Korakis T., Koutsopoulos I., Tassiulas L., Rakotoarivelo T., Ott M., PARMENTELAT T., « Experimentation on end-to-end performance aware algorithms in the federated environment of the heterogeneous PlanetLab and NITOS testbeds », Computer Networks , 04/2014.

[LAC 16] LACHTAR A., VAL T., KACHOURI A., " 3DCane: a monitoring system for the elderly using a connected walking stick », International Journal of Computer Science and Information Security, USA, Vol. 14 nº 8, août 2016.

[LEE 16] LEE H.-H., KWON J.-H., KIM E.-J., « FS-IIoTSim: a flexible and scalable simulation framework for performance evaluation of industrial Internet of things systems », The Journal of Supercomputing, pp 1-18, DOI 10.1007/s11227016-1915-4, November 2016.

[MIB 17] http://mi.iut-blagnac.fr

[MOO 17] MOOC "objets connectés : des radiofréquences aux réseaux, Université de Toulouse, https://www.funmooc.fr/courses/univ-toulouse/101003/session01/about, http://eformation.univ-tlse3.fr/oc/

[NS3 17] https://www.nsnam.org

[ORB 17] ORBIT: Open-Access Research Testbed for Next-Generation Wireless Networks, http://www.orbit-lab.org

[RIO 17] http://riot-os.org

[SHA 17] ShaH A. A., HABIB M., SAJjad T., Umar M., BABAR M., « Applications and Challenges Faced by Internet of Things - A Survey », Future Intelligent Vehicular Technologies, pp.182-188, January 2017.

[SUR 99] SuRYadeVara, N.K., Gaddam A., RAYUdu, R. K. AND Mukhopadhyay, S.C., « Wireless Sensors Network Based Safe Home to Care Elderly People: Behaviour Detection », Proc. Eurosensors XXV, September 4-7, 2011, Athens, Greece, pp. 96-99.

[TEE 17] http://www.pjrc.com/teensy

[VAn 16] Van den Bossche A., Dalce R., Fofana N. I., Val T., « DecaDuino: An Open Framework for Wireless Time-of-Flight Ranging Systems », IFIP Wireless Days (WD 2016), Toulouse, 23/03/2016-25/03/2016

[VDB 16] Van den Bossche A., Campo E., Duchier J., Bougeois E., Blanc Machado M., Val T., Vella F., VIGOUROUX N., "Multidimensional observation methodology for the elderly in an ambient digital environment ", International Conference on Computers Helping People with Special Needs (ICCHP 2016), Linz, Austria, 1315/07/16.

[VEL 13] Vella F., VigourouX N., Boudet B., Rumeau P., " Usage de technologies d'interaction par des personnes âgées atteintes d'une maladie d'Alzheimer », Workshop - Alzheimer, Approche pluridisciplinaire - De la recherche clinique aux avancées technologiques, Toulouse, France, IRIT, p. 123-134, 25/01/2013. 
[Vel 15] Vella F., Sauzin D., Truillet P., Vial A., Vigouroux N., « Codesign of the Medical Assistive and Transactional Technologies system », Recherche en Imagerie et Technologies pour la Santé (RITS 2015), Dourdan, IEEE French Section, p. 122-123,25-27/03/2015.

[VIG 15] Vigouroux N., Rumeau P., Boudet B., Vella F., Salvodelli M., « Wellfar-e-link®: true life lab testing of a homecare communication tool », Non-pharmacological Therapies in Dementia, Nova Science Publishers, Vol. 3 N. 2, p. 133-142, 2015.

[VIT 17] VITAL: The future of Smart Cities. http://vital-iot.eu/, 2017

[WIN 17] http://wino.cc

[WU 08] Wu W., "The SmartCane system: an assistive device for geriatrics », BodyNets'08, ICST 3rd international conference on Body area networks, Article No. 2, Tempe, Arizona, March 13 - 17, 2008. 